\title{
Estimation of Direct Medical Costs Related to Chronic Hepatitis C: A Rationale for Early Antiviral Therapy
}

\author{
Do Young Kim \\ Department of Internal Medicine, Yonsei University College of Medicine, Seoul, Korea
}

See "Healthcare Costs for Chronic Hepatitis C in South Korea from 2009 to 2013: An Analysis of the National Health Insurance Claims' Data" by Moran Ki, et al. on page 835, Vol. 11. No. 6, 2017

In medicine, cost-of-illness studies play a role as they might be used as references for resource allocation, development of policy, and for determination of the cost-effectiveness of new therapies. ${ }^{1}$ Chronic hepatitis C (CHC) is a serious worldwide health-related burden, and recent development and introduction of direct-acting antivirals (DAAs) for CHC has made a substantial push toward looking the disease from the economic point of view. ${ }^{2}$ Economic impact of $\mathrm{CHC}$ and its management can be evaluated by two different approaches. The first is cost-ofillness study, where only immediate costs of treatment for CHC are considered. Whereas, cost-effectiveness study usually needs more assumptions and should include clinical outcomes such as disease related morbidity or mortality. Thus, cost-of-illness studies may provide actual and fundamental data to properly conduct cost-effectiveness studies.

Various methodologies in the area of health economy can be applied to cost-of-illness studies, each having its own advantages and disadvantages. Furthermore, because of divergence in healthcare system and reimbursement policy, it is almost impossible to compare directly the costs incurred by a disease among countries. It is also noteworthy that many of the studies estimate only direct costs related to the disease, excluding indirect costs such as loss of productive job and transportation fees due to absent or incorrect data. ${ }^{3}$

Cost-estimation studies related to $\mathrm{CHC}$ have not been presented in Asian region, even though such countries as Japan and Taiwan have a high prevalence of hepatitis $\mathrm{C}$ virus (HCV) infection. In this regard, the article by Ki et al. ${ }^{4}$ in this issue might be relevant, which examined the annual per-patient costs for CHC, cirrhosis and hepatocellular carcinoma (HCC) using Korean public data. The National Health Insurance (NHI) and Health
Insurance Review and Assessment (HIRA) claim data between 2009 and 2013 were the sources for the investigation. Indeed, the NHI in South Korea is an unique healthcare system, where both costs incurred by inpatient and outpatient care are co-paid by government and patient himself, leaving some items such as practices, drugs and devices still nonreimbursed. The merit of including all the people in Korean national insurance system pushed the ex-president of the U.S., Mr. Obama, to revolve U.S. healthcare system and to make "Patient Protection and Affordable Care Act," the so called "Obama Care." In real practice, however, Korean NHI system has produced lots of abnormal and deviated medical practices due to limited resources, budget and non-optimal insurance costs given to the physicians. Anyhow, the national universal insurance system enabled the authors to perform a comprehensive and uniform cost-of-illness study regarding HCV infection, encompassing all the disease states from hepatitis to HCC, and all the classes of hospitals from private clinic to tertiary university hospital.

In the article of Ki et al., ${ }^{4}$ a total of 181,768 patients were identified during the study period, and the direct annual perpatient medical costs, which were calculated by prevalencebased approach, increased from CHC to cirrhosis, HCC and the first year post-liver transplantation; 895, 1,873, 6,945, and 67,359 USD, respectively. As expected, the direct medical costs increased with progression of $\mathrm{CHC}$, because complications of cirrhosis such as ascites, variceal bleeding or HCC required hospitalization and resource utilization. Increasing direct medical costs per patient with more advanced CHC-related disease were also shown in a previous Korean study, where cost data on 445 CHC patients from eight institutions were retrospectively reviewed and the monthly direct costs per patient in CHC, com-

\footnotetext{
Correspondence to: Do Young Kim

Department of Internal Medicine, Yonsei University College of Medicine, 50-1 Yonsei-ro, Seodaemun-gu, Seoul 03722, Korea

Tel: +82-2-2228-1992, Fax: +82-2-393-6884, E-mail: dyk1025@yuhs.ac

pISSN 1976-2283 eISSN 2005-1212 https://doi.org/10.5009/gnl17408

@ This is an Open Access article distributed under the terms of the Creative Commons Attribution Non-Commercial License (http://creativecommons.org/licenses/by-nc/4.0) which permits unrestricted non-commercial use, distribution, and reproduction in any medium, provided the original work is properly cited.
} 
pensated cirrhosis and HCC were estimated to be 77, 98, and 504 USD, respectively. ${ }^{5}$ Thus, annual costs per patient in each disease state are calculated to be 924, 1,176, and 6,048 USD, respectively, which are similar to the estimates in Ki's study.

The number of patients who received antiviral therapy with interferon (or pegylated interferon) and/or ribavirin at least once was 25,223 accounting for $13.9 \%$ of all the patients between 2009 and 2013. Interestingly, the costs per patient during the study period were 19,743 USD in those who underwent antiviral therapy, while the costs in those who did not undergo antiviral therapy were 3,126 USD. A substantial proportion (78.5\%) of costs in patients who received antiviral therapy was incurred by drugs including pegylated interferon. However, 37.2\% of the total costs were incurred by drugs other than antiviral agents in those who did not undergo antiviral therapy. What should be kept in mind is that cost-of-illness study just estimates immediate costs, and it might be addressed by another kind of cost study whether antiviral treatment-related high costs could lower overall costs in long-term outcomes.

The authors in their study highlighted low rates of anti-HCV treatment uptake (13.9\%) over 5-year period. Though these figures might underestimate the real rates of treatment because some patients had already underwent antiviral therapy before the study period, indeed a significant proportion of CHC patients did not received therapy in the interferon era due to various reasons including advanced liver fibrosis, old age and fear of adverse events. Additional finding related to antiviral therapy is that 1,471 patients received ribavirin monotherapy without interferon or pegylated interferon, which is not a recommended regimen. There is a need of advertisement and education on the proper anti-HCV regimen for physicians in the community utilizing this analysis.

Claim data have its own limitations, resulting in inaccuracy and ambiguity. As the authors stated, it may be argued whether diagnosis of $\mathrm{CHC}$, cirrhosis and HCC was accurately made with ICD-10 codes. Unfortunately, the costs related to management of decompensated cirrhosis were not separately estimated due to this limitation. Exclusion of non-reimbursed costs from the estimation, and the possibility of including costs incurred by comorbidities not by CHC itself are also disadvantages.

It has been apparent that healthcare costs increase if CHCrelated diseases are not properly controlled. Overall, the increase of costs per person for HCC seems to be greater compared to CHC or cirrhosis; from 5,838 to 6,945 USD in HCC versus from 1,470 to 1,873 USD in cirrhosis versus from 813 to 895 USD in CHC (from 2009 to 2013). Therefore, the issue of whether stopping progression of disease with DAAs could decrease healthcare costs needs to be further studied using these data.

\section{CONFLICTS OF INTEREST}

No potential conflict of interest relevant to this article was reported.

\section{REFERENCES}

1. El Khoury AC, Wallace C, Klimack WK, Razavi H. Economic burden of hepatitis C-associated diseases: Europe, Asia Pacific, and the Americas. J Med Econ 2012;15:887-896.

2. Jeong SH, Jang ES, Choi HY, Kim KA, Chung W, Ki M. Current status of hepatitis $\mathrm{C}$ virus infection and countermeasures in South Korea. Epidemiol Health 2017;39:e2017017.

3. Rein DB, Borton J, Liffmann DK, Wittenborn JS. The burden of hepatitis C to the United States Medicare system in 2009: descriptive and economic characteristics. Hepatology 2016;63:11351144.

4. Ki M, Choi HY, Kim KA, Jang ES, Jeong SH. Healthcare costs for chronic hepatitis C in South Korea from 2009 to 2013: an analysis of the National Health Insurance claims' data. Gut Liver 2017;11:835-842.

5. Kim DY, Yoon KT, Kim W, et al. Estimation of direct medical cost related to the management of chronic hepatitis $\mathrm{C}$ and its complications in South Korea. Medicine (Baltimore) 2016;95:e3896. 